\title{
CUESTIONARIO DE ESTRATEGIAS UTILIZADAS POR LOS JÓVENES PARA NEGOCIAR EL USO DEL PRESERVATIVO
}

\author{
Gras, M.E. \\ eugenia.gras@udg.edu \\ Planes, M. \\ Gómez, A.B. \\ Instituto de Investigación sobre Calidad de Vida \\ Universidad de Girona
}

Fecha de Recepción: 14 Febrero 2018

Fecha de Admisión: 10 Abril 2018

\section{RESUMEN}

Se analiza la estructura factorial de un instrumento para evaluar la percepción que tienen los jóvenes sobre la adecuación de trece estrategias para negociar el uso del preservativo. La muestra está formada por 260 estudiantes de grado (81.4\% mujeres, edad media= 21 años; dt=2.8). Las valoraciones se hicieron con una escala del 0 (nada adecuada) al 10 (totalmente adecuada). Los resultados del análisis factorial exploratorio muestran que las trece estrategias se agrupan en tres factores: en un primer factor aparecen aquellas que se refieren a la protección que proporciona el preservativo (frente a infecciones, embarazos, etc.), en un segundo factor aquellas que formulan la petición de forma más o menos directa (pedir su uso, mostrar un preservativo, etc.) y finalmente un tercer factor que engloba aquellas que utilizan argumentos con carga emocional (chantaje, amenazar con no tener relaciones, etc.).

Palabras clave: uso del preservativo; estrategias de negociación; jóvenes

\section{ABSTRACT}

A questionnaire of strategies to negotiate condom use among young people.

We analyze the factorial structure of an instrument to assess young people perception of the adequacy of thirteen strategies to negotiate the use of condoms. The sample consists of 260 undergraduate students $(81.4 \%$ women, mean age $=21$ years, $s d=2.8)$. Assessments were made with a scale from 0 (not adequate) to 10 (totally adequate). The results of the exploratory factor analysis show that the thirteen strategies are grouped into three factors: in a first factor are the strategies based on the protection provided by the condom (against infections, pregnancies, etc.), in a second factor those requesting more or less directly the use of condom (request its use, show a condom, 


\section{CUESTIONARIO DE ESTRATEGIAS UTILIZADAS POR LOS JÓVENES PARA NEGOCIAR EL USO DEL PRESERVATIVO}

etc.) and, finally, a third factor that includes strategies involving emotional-loaded arguments (blackmail, threatening with not having relationships, etc.)

Keywords: condom use; negotiation strategies; youth

\section{ANTECEDENTES:}

El uso del preservativo es la medida preventiva más recomendada para evitar simultáneamente las infecciones de transmisión sexual y los embarazos no deseados (UNAIDS, 2016). Las tasas de uso del preservativo entre los jóvenes son bastante elevadas dependiendo de los colectivos examinados, pero están lejos de ser las deseadas. Folch, Álvarez, Casabona, Brotons y Castellsagué (2015) comunican que en una muestra de 800 jóvenes catalanes, lo han utilizado en su última relación sexual el $75.6 \%$ de los chicos y el $58.4 \%$ de las chicas de entre 16 y 24 años, mientras que Planes, Prat, Gómez, Gras y Font-Mayolas (2012) informan que en una muestra de 1711 estudiantes universitarios, dicen haberlo empleado siempre el $60.2 \%$ de los varones y el $61.7 \%$ de las mujeres.

Si se tiene en cuenta que el uso del preservativo requiere el acuerdo entre los miembros de la pareja, las habilidades de comunicación y de negociación adquieren gran relevancia en el proceso de toma de decisiones.

Desde hace años, diversos autores (De Bro, Campbell y Peplau, 1994; Edgar, Freimuth, Hammond, McDonald y Fink, 1992; Gómez, 2006; Holland y French, 2012; McLaurin-Jones, Lashley y Marshall, 2016; Noar, Morokoff y Harlow, 2002) han recopilado las diferentes estrategias que usan los jóvenes para conseguir utilizar el preservativo con sus parejas, por lo que actualmente poseemos un amplio muestrario de las mismas ("dar información a la pareja sobre riesgo de embarazo", "dar información a la pareja sobre riesgo de infecciones de transmisión sexual", "amenazar a la pareja con no tener relaciones sexuales" "pedir el uso del preservativo de manera clara y concisa", "advertir a la pareja sobre los efectos negativos que tendría sobre la relación afectiva no estar de acuerdo en usar el preservativo", "enseñar un preservativo a la pareja", "poner el preservativo de manera erótica y seductora", etc.).

La revisión de las anteriores estrategias hace pensar que algunas de ellas comparten determinadas características que posiblemente permitan agruparlas en diferentes tipologías o factores.

\section{OBJETIVO:}

El objetivo de este trabajo es analizar la estructura factorial de un instrumento que evalúa la percepción que tienen los jóvenes sobre la adecuación de trece estrategias para proponer el uso del preservativo en una relación sexual con una pareja con la que existen vínculos afectivos.

\section{PARTICIPANTES:}

Participan en el estudio 260 estudiantes de primero, segundo, tercero y cuarto curso del grado de Psicología de la Universidad de Girona que asistieron a clase el día de la recogida de datos. El $81.4 \%$ de los participantes son mujeres y el rango de edad es de 18 a 31 años (edad media $=21$ años; $d t=2.8$ años). Todos los estudiantes presentes en la clase dieron su consentimiento para responder el cuestionario.

\section{METODOLOGÍA:}

\section{Instrumento:}

Se utiliza un cuestionario de estrategias para proponer el uso del preservativo a una pareja con la que existen vínculos afectivos. Dicho cuestionario consta de 13 ítems que se presentan en la tabla 1, extraídos de los estudios revisados en el apartado de Antecedentes. 
Tabla 1. Ítems del Cuestionario de Estrategias de negociación del uso del preservativo

1.Petición directa de utilización del preservativo

2. Aludir a los riesgos para la salud si no se utiliza el preservativo

3. Aludir a la protección que proporciona el preservativo ante las infecciones de transmisión sexual (ITS)

4. Referirse al riesgo de un embarazo no deseado si no se utiliza el preservativo

5. Amenazar con no tener relaciones si no se utiliza el preservativo

6. Pedir a la pareja si tiene un preservativo para utilizarlo

7. Comentar que con preservativo se está más tranquilo y se puede disfrutar más de la relación

8. Argumentar que "si me quieres respetarás que quiera utilizar un preservativo"

9. Argumentar que hay que utilizar el preservativo por no ser aún una pareja estable

10. Decir claramente a la pareja que si no se utiliza el preservativo no tendréis sexo

11. Darle un preservativo a la pareja

12. Poner el preservativo de manera erótica y seductora

13. Argumentar que utilizar el preservativo indica responsabilidad y respeto por la pareja

Los participantes debían valorar en una escala de 0 (nada adecuada) a 10 (totalmente adecuada) en qué medida creían que era adecuada cada una de estas estrategias para proponer el uso del preservativo.

\section{Procedimiento:}

Los datos se recogieron en los últimos minutos de clase de una asignatura obligatoria tras pedir autorización a los profesores responsables. Se pidió el consentimiento a los estudiantes para participar en el estudio y se garantizó el anonimato de las respuestas y el uso de los resultados solo con fines de investigación. El tiempo aproximado de respuesta del cuestionario fue de 10 minutos.

\section{Análisis de datos:}

Para el análisis de los datos se utilizó el análisis factorial exploratorio con el método de extracción de componentes principales y rotación varimax. Se utilizó el test de esfericidad de Barlett para analizar las interrelaciones entre variables y el índice Kaiser-Meyer-Olkin como medida de adecuación del tamaño de la muestra. Para determinar el número de factores a extraer se usó el gráfico de sedimentación. Para evaluar la consistencia interna de los ítems se utilizó el coeficiente alfa de Cronbach. Todos los datos se analizaron con el paquete estadístico SPSS V. 23.

\section{RESULTADOS:}

El análisis previo de los ítems muestra que están intercorrelacionados $\left(X^{2}\right.$ de Bartlett $=766.07$; $p<.001$ ) y que la medida de la muestra es adecuada (KM0 $=0.73$ ), por lo que es procedente realizar el análisis factorial. Observando el gráfico de sedimentación se opta por una solución de tres factores. 
En el primer factor cargan los ítems 2, 3 y 4. Dichos ítems están relacionados con la protección que ofrece el preservativo ante las infecciones de transmisión sexual, los embarazos no deseados y otros riesgos para la salud. En el segundo factor cargan seis ítems $(1,6,7,11,12$ y 13$)$ relacionados con la petición a la pareja del uso del preservativo, realizada de modo más o menos directo tales como pedirlo o darlo o empleando argumentos no relacionados con la salud (por ejemplo: se está más tranquilo y se puede disfrutar más de la relación). El tercer factor engloba cuatro estrategias (ítems 5, 8, 9 y 10) que presentan argumentos/advertencias con carga emocional negativa tales como chantaje ("si me quieres respetarás que quiera utilizar el preservativo") 0 amenazas de no tener relaciones sexuales en caso de no querer utilizarlo.

En la tabla 2 se presentan las cargas factoriales de todos los ítems ordenadas por factores y de mayor a menor y los índices alfa de Cronbach correspondientes. Todas las cargas factoriales son iguales o superiores a 0.49 y ningún ítem tiene carga en dos factores superior a 0.34 . Los valores alfa oscilan entre 0.64 y 0.86 e indican que existe una consistencia interna aceptable entre los ítems de cada factor. Los tres factores explican un $52.84 \%$ de la variancia total.

Tabla 2. Cargas factoriales, índices de consistencia interna y variancia rotada

\begin{tabular}{|c|c|c|c|}
\hline & Factor 1 & Factor 2 & Factor 3 \\
\hline \multicolumn{4}{|l|}{$\begin{array}{l}\text { Factor } 1 . \text { Protección de la salud. } \\
\text { Alfa de } \text { Cronbach }=0.86 . \% \text { Variancia }=18.68\end{array}$} \\
\hline 3. Protección ante las ITS & 0.90 & & \\
\hline 2. Riesgos para la salud & 0.89 & & \\
\hline 4. Riesgo de embarazo & 0.79 & & \\
\hline \multicolumn{4}{|l|}{$\begin{array}{l}\text { Factor 2. Petición o argumento no relacionado con } \\
\text { la salud. } \\
\text { Alfa de Cronbach }=0.64 . \% \text { Variancia }=17.90\end{array}$} \\
\hline 11. Darle un preservativo & & 0.78 & \\
\hline 6. Pedirle un preservativo & & 0.66 & \\
\hline 1. Petición directa & & 0.56 & \\
\hline 13. Responsabilidad y respeto por la pareja & & 0.52 & \\
\hline 7. Se puede disfrutar más de la relación & & 0.50 & \\
\hline 12. Ponerle el preservativo & & 0.49 & \\
\hline \multicolumn{4}{|l|}{$\begin{array}{l}\text { Factor 3: Argumento/advertencia con carga } \\
\text { emocional negativa. } \\
\text { Alfa de Cronbach }=\mathbf{0 . 6 9} \% \text { Variancia }=\mathbf{1 7 . 0 7}\end{array}$} \\
\hline 5. Amenazar con no tener relaciones & & & 0.73 \\
\hline 8. Si me quieres respetarás que quiera utilizarlo & & & 0.72 \\
\hline 9. No somos pareja estable & & & 0.68 \\
\hline 10. Sin preservativo no hay sexo & & & 0.66 \\
\hline
\end{tabular}




\section{DISCUSIÓN}

El cuestionario de estrategias para negociar el uso del preservativo presenta una estructura factorial de 3 factores que reflejan tres modos diferentes de proponer su uso: 1/ utilizando argumentos de protección contra infecciones de transmisión sexual o de evitación de riesgos para la salud y de embarazos no deseados, 2/ haciendo una petición más 0 menos directa 0 con argumentos no relacionados con la protección de la salud y 3 / utilizando argumentos/advertencias con carga emocional negativa (chantaje, amenazas, etc.). La consistencia interna entre los ítems de cada factor es aceptable.

Nuestros resultados coinciden en gran medida con los comunicados por Tschann, Flores, de Groat, Deardorff y Wibbelsman (2010) con una muestra de 694 jóvenes. Los autores obtuvieron 3 factores a partir de 15 estrategias utilizadas para lograr el uso del preservativo, a los cuales llamaron "información sobre el riesgo" "comunicación directa verbal y no verbal" e "insistencia". Existe una gran coincidencia entre los dos primeros factores de ambos estudios. Nuestro tercer factor "argumentación/advertencia con carga emocional negativa" también apareció en el estudio citado como resultado del análisis factorial de 15 estrategias que empleaban los jóvenes para no utilizar el preservativo.

También se asemejan a los obtenidos por Hanna (1999) con una muestra de 209 jóvenes, utilizando un instrumento con 25 ítems que incluían aspectos que facilitaban o dificultaban el uso del preservativo. Los ítems se estructuraron en tres factores y, aunque con distinta nomenclatura a la utilizada en nuestro estudio, en dos de ellos -el primero y el segundo- se observan similitudes. El primer factor comprende ítems relativos al acto de pedir el uso del preservativo y el segundo a la capacidad del preservativo para proteger la salud propia y la de la pareja; mientras que el tercero incluye diversas reflexiones sobre la reducción del placer sexual, que no se contemplan en nuestro instrumento.

Este estudio tiene una serie de limitaciones. La principal está relacionada con la selección de la muestra que no permite la generalización a otros colectivos de jóvenes ni a la población general. Sería aconsejable aplicar el cuestionario a otras muestras de jóvenes más amplias y utilizar el análisis factorial confirmatorio para validar el modelo de tres factores.

También seria de mucho interés comprobar la capacidad predictiva de uso del preservativo, de los diferentes factores que forman parte del cuestionario.

\section{CONCLUSIONES}

Se identifican tres tipos de estrategias de negociación del preservativo con una pareja con la que se tienen vínculos afectivos: las relacionadas con la protección de la salud, la petición directa 0 con argumentos no relacionados con la salud y los argumentos/ advertencias con carga emocional negativa.

Las estrategias relacionadas con la protección de la salud contienen información ampliamente difundida y aceptada entre los jóvenes. La petición directa o con argumentos no relacionados con la salud incluye tanto conducta verbal como no verbal. Las advertencias con carga emocional negativa incluyen actuaciones que pueden dañar las relaciones interpersonales, al margen de que consigan ser efectivas en determinados momentos.

El cuestionario puede ser útil para detectar estrategias poco adecuadas en la negociación del uso del preservativo y, así mismo, para evaluar el efecto de programas formativos que incidan sobre las habilidades de negociación del uso del preservativo. 


\section{REFERENCIAS}

DeBro, S., Campbell, S. y Peplau, L. (1994). Influencing a partner to use a condom. A college student perspective. Psychology of Women Quarterely, 18, 165 - 182.

Edgar, T., Freimuth, V., Hammond, S., McDonald, D. y Fink, E. (1992). Strategic sexual communication: condom use resistance and response. Health Communication, 4 (2), $83-104$.

Folch, C., Álvarez, J. L., Casabona, J., Brotons, M., Castellsagué, X. y Grupo Jóvenes e Internet (2015). Determinantes de las conductas sexuales de riesgo en jóvenes de Cataluña. Revista Española de Salud Pública, 89: 471-485.

Gomez, A.B. (2006). Negociacion del uso del preservativo con una pareja afectiva reciente: expectativas de autoeficacia y habilidades de comunicacion (Trabajo de investigacion de doctorado no publicado). Universitat de Girona.

Hanna, K.M. (1999). An adolescent and young adult condom perception scale. Western Journal of Nursing Research, 21, 621-634.

Holland, K.J. y French, S.E. (2012). Condom negotiation strategy use and effectiveness among college students. Journal of Sex Research, 49, 443-453.

McLaurin-Jones,T., Lashley,M., y Marshall, V. (2016). Minority college women's views on condom negotiation. International Journal of Environmental Research and Public Health, 13, 40-52.

Noar, S.M., Morokoff, P.J. y Harlow, L. (2002). Condom negotiation in heterosexually active men and women: the development and validation of a condom influence strategy questionnaire. Psychology and Health, 17(6), 711-735.

Planes, M., Prat, F.X, Gómez, A.B., Gras, M.E. i Font-Mayolas, S. (2012). Ventajas e inconvenientes del uso del preservativo con una pareja afectiva heterosexual. Anales de Psicología, 28 (1), 161170.

Tschann, J.M., Flores, E., de Groat, C.L, Deardorff, J. y Wibbeisman, Ch.J. (2010). Condom negotiation strategies and actual condom use among latino youth. Journal of Adolescent Health, 47 (3), 254-262.

UNAIDS (2007). Condoms. Recuperado el 21 de abril de 2018 de http://www.unaids/resources/presscentre/.../20161002_condoms. 